CASE REPORT

Volume 16 Issue 22021

DOI: 10.21315/aos2021.16.2.15

ARTICLE INFO

Submitted: $12 / 05 / 2021$

Accepted: 11/08/2021

Online: 22/12/2021

\section{Kimura Disease as a Rare Cause of Proptosis: A Case Report}

\author{
V Sha Kri Eh Dama, Irfan Mohamada ${ }^{a}$, Evelyn Li Min Tai ${ }^{b}$, Adil Hussein ${ }^{b}$, \\ Khairil Amir Sayutic, Fatihatul Munirah Amiruddind, Faezahtul \\ Arbaeyah Hussain $^{d}$
}
${ }^{a}$ Department of Otorhinolaryngology-Head and Neck Surgery, School of Medical Sciences, Universiti Sains Malaysia, 16150 Kota Bharu, Kelantan, Malaysia
${ }^{b}$ Department of Ophthalmology, School of Medical Sciences, Universiti Sains Malaysia, 16150 Kota Bharu, Kelantan, Malaysia

${ }^{c}$ Department of Radiology, School of Medical Sciences, Universiti Sains Malaysia, 16150 Kota Bharu, Kelantan, Malaysia

${ }^{d}$ Department of Pathology, School of Medical Sciences, Universiti Sains Malaysia, 16150 Kota Bharu, Kelantan, Malaysia

${ }^{\star}$ Corresponding author: irfankb@usm.my

To cite this article: Eh Dam VSK, Mohamad I, Tai ELM, Hussein A, Sayuti KA, Amiruddin FM, Hussain FA (2021). Kimura disease as a rare cause of proptosis: A case report. Arch Orofac Sci, 16(2): 259-265. https://doi.org/10.21315/aos2021.16.2.15

To link to this article: https://doi.org/10.21315/aos2021.16.2.15

\begin{abstract}
Kimura disease $(\mathrm{KD})$ is a rare chronic inflammatory disorder of unknown aetiology that primarily affects the head and neck region with lymph node involvement. Young to middle-aged adult Asian males are predominantly affected. The most common presentation is painless subcutaneous swelling in the head and neck region, while proptosis or orbital involvement is very rarely reported. KD shares some features with other inflammatory and neoplastic disorders, including lymphoma; thus, investigations to confirm the diagnosis should not be delayed. Systemic corticosteroids are commonly used to treat KD and show an excellent response; however, the optimal treatment is still uncertain, and $\mathrm{KD}$ has a high recurrence rate. We describe the case of a patient with $\mathrm{KD}$ who presented with proptosis and post-auricular swelling, which responded well to oral prednisolone treatment.
\end{abstract}

Keywords: Chronic inflammatory disorder; corticosteroid; Kimura disease; post-auricular swelling; proptosis

\section{INTRODUCTION}

Kimura disease (KD) is a rare chronic inflammatory disorder of unknown cause that mainly affects the head and neck region (Zhang \& Jiao, 2019). It was first described in 1937 but became widely recognised as Kimura disease in 1948 after the definitive histologic description was published (Kim \& Szeto, 1937; Kimura et al., 1948; Li et al., 1996). It predominantly affects Asian males during the second to fourth decades of life (Zhang \& Jiao, 2019). Classically, KD presents with painless subcutaneous swelling in the head and neck region and predominantly with lymph node involvement (Fouda et al., 2011). Orbital involvement is very rare, with only 14 cases were reported (Takenaka et al., 1976; Azman et al., 2017). 


\section{CASE REPORT}

A 39-year-old male presented with right eye protrusion of six months' duration, which he noticed while taking a photograph of himself. The mass gradually increased in size and was associated with double vision for a month prior to presentation. The patient's symptoms included irritability and fatigue, although he denied other symptoms of hyperthyroidism. There was no history of prolonged cough, fever, loss of appetite or weight loss, and the patient denied tuberculosis contact; however, he was a chronic smoker. He had experienced painless left post-auricular swelling for one year and right pre-auricular swelling for two weeks. There were no ear, nose, or throat symptoms.

Upon examination, the visual acuity was 6/6 bilaterally, without a relative afferent pupillary defect. The right eye showed proptosis with chemosis and lid retraction, but there were no other signs of thyroid ophthalmopathy. The right eye extraocular muscle movements were mechanically limited in abduction and elevation and associated with binocular diplopia in the right gaze and the up gaze. This was confirmed by a Hess test. Other anterior and posterior segment examinations of the eye were normal. Systemic examination revealed swelling at the left post-auricular region that measured $4 \mathrm{~cm} \times 3 \mathrm{~cm}$ and was firm in consistency, mobile and non-tender. Another swelling at the right pre-auricular region measured $1 \mathrm{~cm} \times 1 \mathrm{~cm}$ and was also firm in consistency, mobile and non-tender. Other otorhinolaryngology examinations were unremarkable.

The renal profile and thyroid function tests were normal. The full blood picture showed eosinophilia, no blasts or abnormal mononuclear cells and no left shift. Computed tomography (CT) of the brain/orbit and neck revealed a bulky and enhanced belly of the right inferior rectus muscle measuring $3.4 \mathrm{~cm} \times 2.5 \mathrm{~cm} \times$ $1.7 \mathrm{~cm}$, causing right ocular proptosis. There was involvement of the anterior tendinous insertion. The right intraconal fat was streaky and the ipsilateral lacrimal gland appeared relatively bulky and enhanced. The other extraocular muscles and the optic nerve were normal. The contralateral orbit and cavernous sinus were also normal. A welldefined enhancing lesion was seen in the left post-auricular region measuring $3.9 \mathrm{~cm}$ $\times 1.4 \mathrm{~cm}$ (see Fig. 1). The thyroid lobes were homogenously enhanced, with no focal lesion. Multiple subcentimeter lymph nodes were present at levels Ia, II, III, IV and V. The overall features of the right orbit were suggestive of inflammatory pseudotumour.

Fine needle aspiration cytology of the left post-auricular swelling was performed but provided insufficient tissue to confirm the diagnosis. Thus, an incision biopsy was performed. The histopathology examination results showed features consistent with $\mathrm{KD}$ (see Fig. 2). Hodgkin lymphoma (HL), Langerhan cell histiocytosis (LCH) and systemic mastocytosis were excluded with the negative immunohistochemistry staining of CD30, CD1a and CD117, respectively.

While awaiting the results of the investigations, the patient developed increasing proptosis and chemosis, requiring a lateral canthoplasty (Fig. 3). After establishing a definitive diagnosis of $\mathrm{KD}$, the patient was treated with oral prednisolone $1 \mathrm{mg} / \mathrm{kg} /$ day, to which he showed an excellent response. The systemic corticosteroids were tapered at a rate of $10 \mathrm{mg}$ weekly, and the patient's proptosis completely resolved after a few weeks (see Fig. 4). Extraocular movements were full and Hertel exophthalmometry was equal $(17 \mathrm{~mm})$ bilaterally. His vision remained $6 / 6$ in both eyes, with normal optic nerve function.

\section{DISCUSSION}

Proptosis is described as abnormal protrusion of the eyeball that has varied aetiology, including neoplastic, vascular, traumatic, 


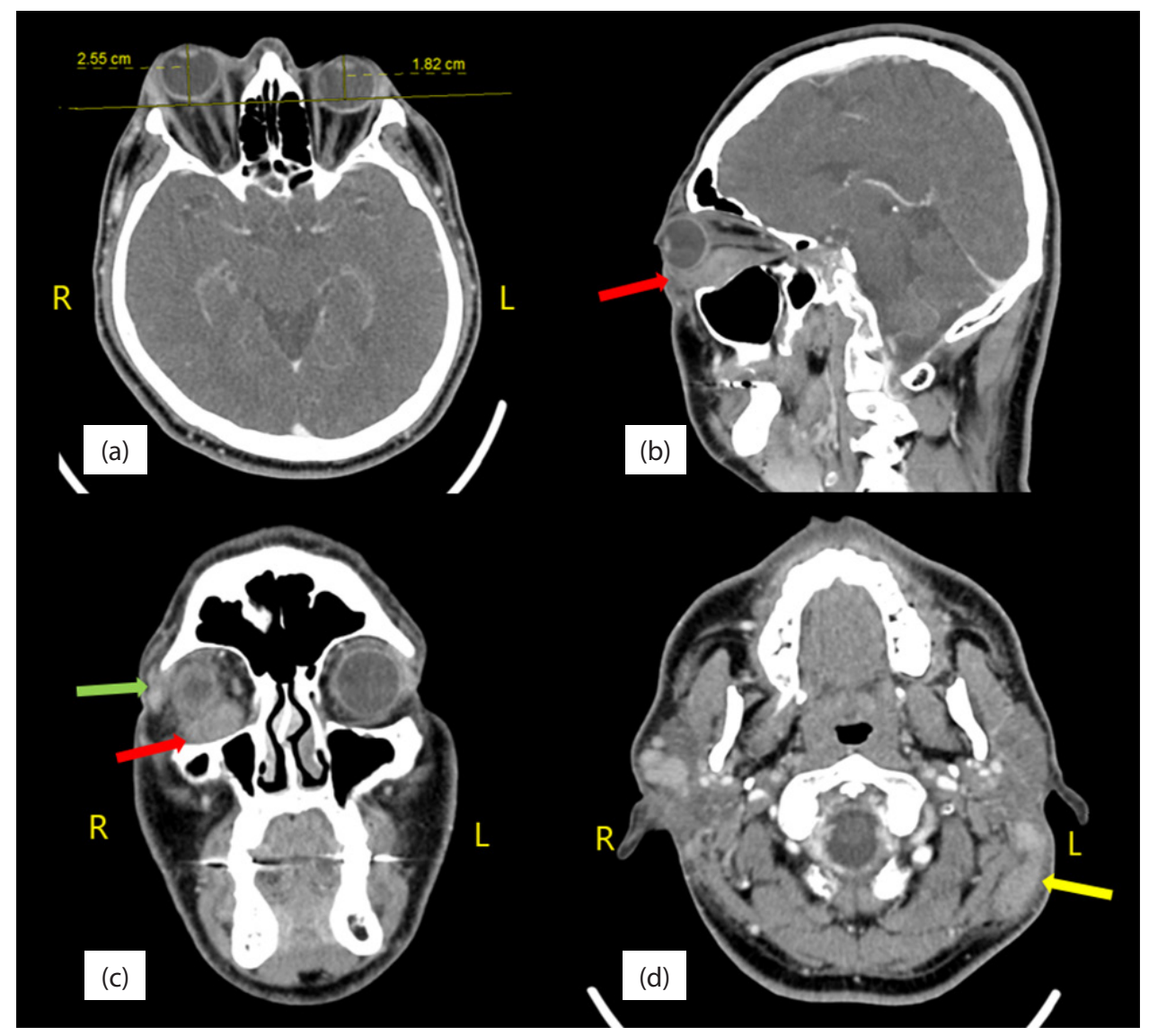

Fig. 1 Contrast enhanced CT at the level of neuro-ocular plane (a) demonstrates the presence of right eye proptosis and right intraconal fat streakiness. The sagittal (b) and coronal (c) views reveal bulky and enhanced belly of the right inferior rectus muscle with involvement of its anterior tendon insertion (red arrow). The right lacrimal gland (green arrow) is also relatively bulky and enhanced. (d) An enhancing lesion is noted at the left post-auricular region (yellow arrow). R: right; L: left.

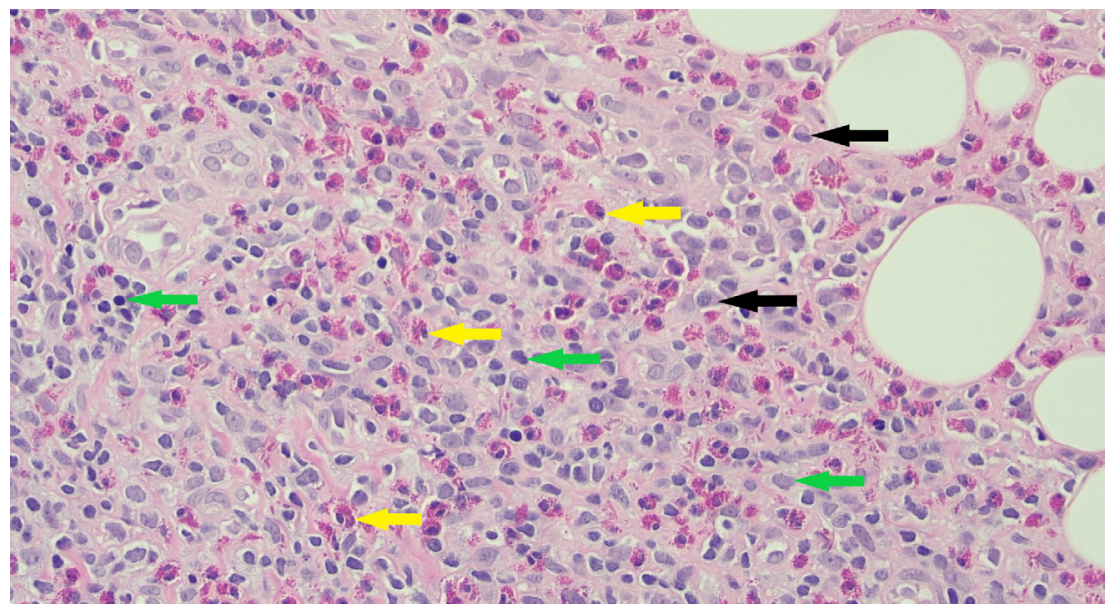

Fig. 2 The lymph node showed many scattered eosinophils (yellow arrows) in the background of reactive and mixed population of lymphocytes (green arrows) and plasma cells (black arrows) (H\&E $\times 400$ ). 


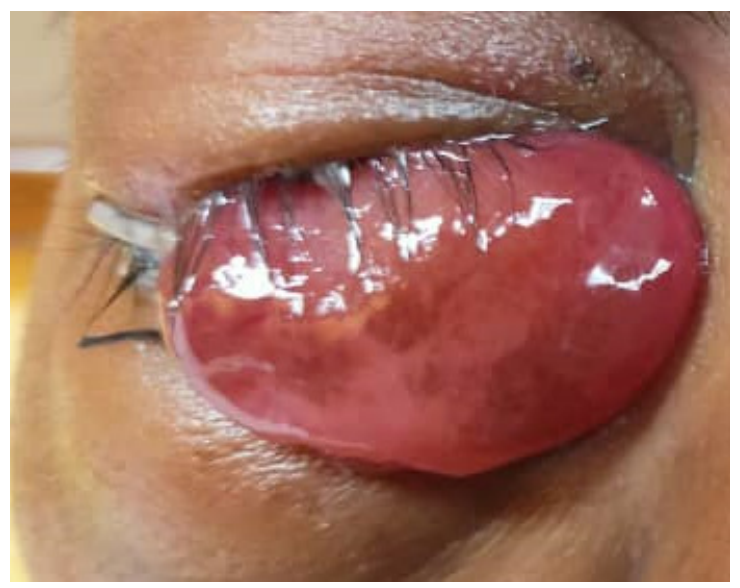

Fig. 3 Right eye worsening chemosis despite a lateral canthotomy.

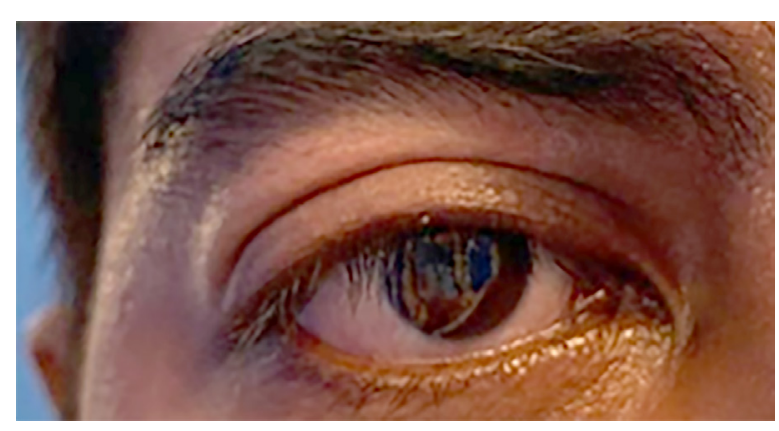

Fig. 4 Right proptosis completely resolved after systemic corticosteroid.

metabolic, infective and inflammatory origins (George \& Nanu, 2016). It is usually classified as unilateral or bilateral to narrow down the differential diagnosis. Chronicity, absence of pain and associated cervical lymphadenopathy are red flags for exclusion of malignancy. The possible initial differential diagnoses of the present case were orbital lymphoma, orbital tuberculosis, sinonasal malignancy and chronic inflammatory disorder.

$\mathrm{KD}$ is a benign chronic inflammatory disease that may have similar features to other inflammatory and neoplastic disorders, such as angiolymphoid hyperplasia with eosinophilia, HL, angioimmunoblastic T-cell lymphoma, allergic granuloma, LCH, Castleman disease and immunoglobulin G4related disease (Zhang \& Jiao, 2019). KD is a rare condition, with fewer than 300 cases reported worldwide up to 1997 (Sun et al., 2008; Bonfils et al., 2013; Dhingra et al., 2019). Asian males are mostly affected, with sporadic cases reported in Europe and the United States (Chen et al., 2004). The male to female ratio ranges from $3.5: 1$ to $6.7: 1$ (Zhang \& Jiao, 2019).

KD usually presents with a subcutaneous mass in the head and neck region, commonly involving the regional lymph node or salivary gland (Dhingra et al., 2019). The parotid region is the most common primary site, followed by the lacrimal gland and the submandibular, axillary, elbow and inguinal regions (Monzen et al., 2014). Orbital involvement is rare, but when it occurs, it usually affects the eyelid or the lacrimal gland. Extraocular muscle involvement is even rarer and can be unilateral or bilateral (Kanazawa et al., 1999; Gonçalves et al., 2016). Right lacrimal gland and inferior rectus muscle were involved in the present case. The most common presentations of $\mathrm{KD}$ with orbital involvement are proptosis, followed by lid swelling, abnormal motility, periocular mass and diplopia (Buggage et al., 1999).

The aetiology and pathophysiology of $\mathrm{KD}$ remain unclear. Allergies or an autoimmune response triggered by some unknown persistent antigenic stimuli have been implicated in its pathophysiology (Dhingra et al., 2019). Blood investigations show raised serum eosinophil counts and immunoglobulin levels, as seen in our case (Gonçalves et al., 2016). The absence of atypical lymphocytes and a left shift in the present case were markers that excluded the possibility of lymphoma.

Imaging with ultrasound, CT or MRI is non-specific for KD. Generally, the presence of multiple ill-defined enhancing masses within and around the parotid gland, with associated regional lymphadenopathy seen with a CT or MRI scan, is suggestive of head and neck KD (Park et al., 2012). In cases with orbital involvement, CT scans 
typically show orbital soft-tissue density, proptosis and variable swelling of the rectus muscles (Buggage et al., 1999). The rectus muscle involvement is usually unilateral, with extension to its tendinous insertion. The common differential diagnosis based on imaging is thyroid-associated orbitopathy, with sparing of the tendinous insertions.

$\mathrm{KD}$ is diagnosed based on clinicopathological features, although there is no pathognomonic feature histologically (Zhang \& Jiao, 2019). Tissue biopsy is the gold standard not only to confirm the diagnosis but also to exclude life-threatening malignancies like lymphoma. Routine initial investigations, such as fine needle aspiration cytology, may not be able to yield a diagnosis due to the limited amount of tissue obtained for examination. Dense fibrosis, capillary proliferation, a mixed population of lymphocytes, reactive lymphoid follicles, absence of Hodgkin cells and diffusely scattered eosinophils are amongst the features suggestive of $\mathrm{KD}$ histologically.

In cases solely involving the eye without other head and neck region involvement, orbital pseudotumour is an important differential diagnosis that shares many similarities with $\mathrm{KD}$, as both diseases are inflammatory in origin. Orbital pseudotumour, also known as idiopathic orbital inflammation, is a benign inflammatory condition of unknown aetiology that can present with acute, subacute or chronic periorbital swelling and predominantly unilateral involvement in the fifth decade of life with no sex predilection (Yeşiltaş \& Gündüz, 2018). The lacrimal glands and extraocular muscles and tendons are the predominant sites of orbital pseudotumour (Kamili et al., 2009), but they were also seen in our patient. Histologically, orbital pseudotumours also show features of non-specific chronic inflammatory infiltrate that may mimic KD features. Furthermore, treatment is relatively similar to that for $\mathrm{KD}$, with systemic corticosteroids being the main modality, while radiotherapy and chemotherapeutic agents are alternatives
(Kamili et al., 2009; Yeşiltaş \& Gündüz, 2018). The reported recurrence rate of orbital pseudotumour is also high.

Although an empirical course of corticosteroids while awaiting the histopathological results was a consideration, we decided against this, as it has been proposed to cause diagnostic delay in malignancy (Gavard-Perret et al., 2015; Mombaerts et al., 2017). Prior to initiating systemic immunosuppressive therapy, it is essential to screen for contraindications, including history taking for tuberculosis, basic metabolic profile, including blood pressure, fasting blood sugar and lipids and a full blood count for occult infection.

Surgical excision, cytotoxic drugs and radiation therapy are alternative treatment modalities. The optimal treatment is still uncertain and a high recurrence rate has been reported (60\% to $80 \%$ ) (Buggage et al., 1999; Zhang \& Jiao, 2019). The dramatic response to corticosteroids in $\mathrm{KD}$ is evidence of the underlying inflammatory nature of the disease (Gonçalves et al., 2016; Azman et al., 2017).

\section{CONCLUSION}

$\mathrm{KD}$ with orbital involvement is very rare, but when it occurs, proptosis is the most common presentation. The clinical features that mimic malignancy, infection and other inflammatory disorders, making the diagnosis of this disease entity very challenging. $\mathrm{KD}$ is diagnosed based on the clinicopathological features, thus tissue biopsy should be performed without delay whenever possible. Oral corticosteroid, surgical excision, cytotoxic drugs and radiation therapy are among treatment modalities; however, the optimal treatment is still uncertain with high recurrence rate. 


\section{REFERENCES}

Azman MS, Jusoh S, Zamli AH, Abd Rahman A (2017). Kimura's disease: Uncommon cause of proptosis. Med Rep Case Stud, 2(2): 1000132. https://doi.org/10.4172/ 2572-5130.1000132

Bonfils P, Moya-Plana A, Badoual C, Nadéri S, Malinvaud D, Laccourreye O (2013). Intraparotid Kimura disease. Eur Ann Otorhinolaryngol Head Neck Dis, 130(2): 87-89. https://doi.org/10.1016/j.anorl.2012 .05 .001

Buggage RR, Spraul CW, Wojno TH, Grossniklaus HE (1999). Kimura disease of the orbit and ocular adnexa. Surv Ophthalmol, 44(1): 79-91. https://doi.org/ 10.1016/s0039-6257(99)00064-8

Chen H, Thompson LD, Aguilera NS, Abbondanzo SL (2004). Kimura disease: A clinicopathologic study of 21 cases. $\mathrm{Am}$ f Surg Pathol, 28(4): 505-513. https://doi .org/10.1097/00000478-200404000-00010

Dhingra H, Nagpal R, Baliyan A, Alva SR (2019). Kimura disease: Case report and brief review of literature. Med Pharm Rep, 92(2): 195-199. https://doi.org/10.15386/ cjmed-1030

Fouda MA, Gheith O, Refaie A, El-Saeed M, Bakr A, Wafa E et al. (2011). Kimura disease: A case report and review of the literature with a new management protocol. Int $f$ Nephrol, 2010: 673908. https://doi .org/10.4061/2010/673908

Gavard-Perret A, Lagier J, Delmas J, Delas J, Adenis JP, Robert PY (2015). [Rationale for a diagnostic approach in non-Graves' orbital inflammation - Report of 61 patients]. F Fr Ophtalmol, 38(10): 912-923. https://doi.org/10.1016/j.jfo.2015.04.017

George TA, Nanu D (2016). Proptosis: Profile from a tertiary care centre in northern Kerala. Int $\mathcal{f}$ Contemp Med Res, 3(12): 3555-3557.
Gonçalves AC, Moritz RB, Aldred VL, Monteiro ML (2016). Bilateral extraocular muscles enlargement from Kimura's disease of the orbit. Indian f Ophthalmol, 64(7): 538-540. https://doi.org/10.4103/0301-4738.118424

Kamili MA, G A, Dar IH, Dar SH, Wazir HS, Qureishi T (2009). Orbital pseudotumor. Oman f Ophthalmol, 2(2): 96-99. https://doi.org/10.4103/0974-620X.53043

Kanazawa S, Gong H, Kitaoka T, Amemiya $\mathrm{T}$ (1999). Eosinophilic granuloma (Kimura's disease) of the orbit: A case report. Graefes Arch Clin Exp Ophthalmol, 237(6): 518-521. https://doi.org/10.1007/ s004170050272

Kim HT, Szeto C (1937). Eosinophilic hyperplastic lymphogranuloma, comparison with Mikulicz's disease. Chin Med F, 23: 699-700.

Kimura T, Voshimura S, Ishikawa E (1948). On the unusual granulation combined with hyperplastic changes of lymphatic tissues. Trans Soc Pathol Fpn, 37: 170-180.

Li TJ, Chen XM, Wang SZ, Fan MW, Semba I, Kitano M (1996). Kimura's disease: A clinicopathologic study of 54 Chinese patients. Oral Surg Oral Med Oral Pathol Oral Radiol Endod, 82(5): 549-555. https://doi.org/10.1016/s1079-2104(96) 80202-2

Mombaerts I, Bilyk JR, Rose GE, McNab AA, Fay A, Dolman PJ et al. (2017). Consensus on diagnostic criteria of idiopathic orbital inflammation using a modified Delphi approach. $\mathcal{F A M A}$ Ophthalmol, 135(7): 769-776. https://doi.org/10.1001/ jamaophthalmol.2017.1581

Monzen Y, Kiya K, Nishisaka T (2014). Kimura's disease of the orbit successfully treated with radiotherapy alone: A case report. Case Rep Ophthalmol, 5(1): 87-91. https://doi.org/10.1159/000360789 
Park SW, Kim HJ, Sung KJ, Lee JH, Park IS (2012). Kimura disease: CT and MR imaging findings. AfNR Am $\mathcal{F}$ Neuroradiol, 33(4): 784-788. https://doi.org/10.3174/ ajnr.A2854

Sun QF, Xu DZ, Pan SH, Ding JG, Xue ZQ, Miao CS et al. (2008). Kimura disease: Review of the literature. Intern Med f, 38(8): 668-672. https://doi.org/10.1111/j $.1445-5994.2008 .01711 . x$

Takenaka T, Okuda M, Usami A, Kawabori S, Ogami Y (1976). Histological and immunological studies on eosinophilic granuloma of soft tissue, so-called Kimura's disease. Clin Allergy, 6(1): 27-39. https://doi.org/10.1111/j.1365-2222.1976 .tb01409.x
Yeșiltaș YS, Gündüz AK (2018). Idiopathic orbital inflammation: Review of literature and new advances. Middle East Afr $\mathcal{f}$ Ophthalmol, 25(2): 71-80. https://doi.org/ 10.4103/meajo.MEAJO_44_18

Zhang X, Jiao Y (2019). The clinicopathological characteristics of Kimura disease in Chinese patients. Clin Rheumatol, 38(12): 3661-3667. https://doi.org/10.1007/s10067 $-019-04752-6$ 